\title{
Effect of pregnane $X$ receptor polymorphisms on tacrolimus blood concentrations and the resulting adverse reactions in kidney transplantation recipients
}

\author{
Z.P. Wang ${ }^{1 *}$, M. Zhao ${ }^{2 *}$, Q.S. $\mathbf{Q u}^{1}$ and S.Z. Miao ${ }^{1}$ \\ ${ }^{1}$ Renal Transplantation Department of Zhengzhou People's Hospital, \\ Zhengzhou, Henan, China \\ ${ }^{2}$ Zhengzhou Shuqing Medical College, Zhengzhou, Henan, China \\ *These authors contributed equally to this study. \\ Corresponding author: Z.P. Wang \\ E-mail: wangzhenpu11@163.com
}

Genet. Mol. Res. 15 (3): gmr.15038464

Received January 20, 2016

Accepted May 12, 2016

Published September 16, 2016

DOI http://dx.doi.org/10.4238/gmr.15038464

Copyright $(C 2016$ The Authors. This is an open-access article distributed under the terms of the Creative Commons Attribution ShareAlike (CC BY-SA) 4.0 License.

\begin{abstract}
We investigated the effect of pregnane $X$ receptor (PXR) polymorphisms on tacrolimus (FK506) blood trough concentrations and the associated adverse reactions in kidney transplantation recipients (KTRs). Polymerase chain reaction (PCR)-restriction fragment length polymorphism was used to detect the genotypes of single nucleotide polymorphism loci in 336 KTRs. The PXR six-base deletion mutation was classified using specific allele PCR, and the FK506 blood trough concentration in the KTRs was measured by chemiluminescent microparticle immunoassay. There were significant differences in adverse reactions resulting from FK506 in age, weight, body mass index $(\mathrm{BMI})$ and treatment course $(\mathrm{P}<0.05)$. Logistical regression
\end{abstract}


revealed that the FK506 treatment course and BMI were risk factors for hyperlipidemia, and the risk of hyperlipidemia increased 27.534 times when the BMI was less than 18.5. Moreover, age was also a risk factor leading to hyperglycemia. FK506 blood trough concentration and $\mathrm{C}_{0} / \mathrm{D}$ value had an impact on adverse reactions induced by hyperglycemia. The KTRs' PXR rs3842689, rs6785049, and rs1523127 mutation frequencies were $26.07,11.79$, and $16.07 \%$, respectively. There was no statistically significant difference in the mutation frequency of each locus between the control group and the adverse reaction groups. Therefore, rs3842689, 7635G $>A$ (rs6785049), and 24381C $>A$ (rs1523127) PXR polymorphisms have no obvious impact on FK506; furthermore, the PXR rs3842689 wild-type homozygous WW genotype is a risk factor of FK506 and results in gastrointestinal reactions.

Key words: Pregnane X receptor; Tacrolimus; Kidney transplantation; Adverse reaction; Gene polymorphism

\section{INTRODUCTION}

Tacrolimus (FK506) metabolizes via the liver and intestinal tract, and is a substrate of P-glycoprotein. To date, research on FK506 pharmacogenetics has mainly focused on CYP3A4, CYP3A5, and MDR1 gene polymorphisms (Hesselink et al., 2003; Wang et al., $2015 a, b)$. Pregnane X receptor (PXR), a new-found orphan nuclear receptor, expresses in the liver and partially expresses in the colon and small intestine; it participates in the regulation of substance absorption and metabolism. Miura et al. (2008) reported that the prednisolone area under the curve in PXR $7635 \mathrm{G}$ allele carriers decreases significantly; the PXR A7635G gene polymorphism can also affect the FK506 clearance rate, and the clearance rates in A allele carrier and GG genotype patients are $3.9 \pm 0.3$ and $5.4 \pm 0.6 \mathrm{~L} / \mathrm{h}$, respectively (Press et al., 2009). PXR C25385T is effective in affecting $\mathrm{FK} 506$ apparent clearance $(\mathrm{Cl} / \mathrm{F})$ (Benkali et al., 2009). However, Elens et al. (2007) did not discover pharmacokinetic correlations between A11156C and T11193C and FK506 in liver transplantation recipients.

Therefore, the PXR gene polymorphism may play an important role in FK 506 metabolism. However, race has an effect on individual differences in drug metabolism, curative effects, and adverse reactions; consequently, whether the unknown single nucleotide polymorphism affects FK506 pharmacokinetics in the Chinese population requires further investigation (Fei et al., 2006; Qi et al., 2012; Yuan et al., 2012). In this study, we examined the correlation between PXR gene polymorphisms and FK506 blood trough concentration, and investigated susceptible genetic factors that lead to adverse reactions after kidney transplantation.

\section{MATERIAL AND METHODS}

\section{Research objects}

We selected a total of 336 kidney transplantation recipients (KTRs) (aged 18 to 78 years) who had received transplants in the Zhengzhou People's Hospital between 1998 and 2014, and who were devoid of other serious diseases. The KTRs were willing to take part in

Genetics and Molecular Research 15 (3): gmr.15038464 
the experiment and signed written informed consent. Patients who had failed to follow-up, had received multi-organ transplantation, had suffered from liver or kidney injury before medication, or had been diagnosed with tuberculosis or hepatitis B/C were excluded from this study. This experiment was approved by the Ethics Committee of Zhengzhou People's Hospital.

\section{Dosage regimen}

A trigeminy immunosuppression regimen (FK506 + mycophenolate mofetil (MMF) + prednisone) was administered. Patients were first given $0.15-0.3 \mathrm{mg} / \mathrm{kg}$ immunosuppression therapy and then MMF by oral administration on an empty stomach (1.5-2.0 g/day). The dosage was then gradually decreased to $0.5-1 \mathrm{~g} /$ day. During the operation, the patients were treated with methylprednisolone by intravenous drip ( $300 \mathrm{mg} /$ day). Three days after the operation, the patients took prednisone orally ( $40 \mathrm{mg} /$ day), and the dosage was reduced to $30 \mathrm{mg} /$ day on the 7 th day, followed by $5-10 \mathrm{mg} /$ day to maintain the treatment.

\section{Observational indices}

The subjects were asked to provide data on gender, age, stature, weight, body mass index, transplantation time, time of dialysis, cadaveric kidney donation or related renal transplantation, and history of diabetes, hypertension, or hepatitis $\mathrm{B} / \mathrm{C}$ infection. Daily oral dosages of FK506 at different time points, blood trough concentrations, prednisone and MMF dosages, drug combination data, hepatic and renal function, fasting plasma glucose, blood routine, blood lipid, blood pressure, and other inspection results were also recorded.

\section{Measurement of FK506 blood concentration}

\section{Sample preparation and measurement}

Venous blood $(2 \mathrm{~mL})$ was taken from transplant recipients during the morning following medication administration. After ethylenediaminetetraacetic acid (EDTA) anticoagulation, $200 \mu \mathrm{L}$ blood was mixed for $5 \mathrm{~s}$ and moved into a centrifuge tube with a pipette. The precipitant $(200 \mu \mathrm{L})$ was added, fully mixed for $30 \mathrm{~s}$, and centrifuged for $5 \mathrm{~min}$ at 13,000 $\mathrm{rpm}$. The supernatant was added to an EDTA glass tube and mixed for 2 $\mathrm{s}$, then subjected to fully automated immunochemistry analysis to measure FK506 trough concentration $\left(\mathrm{C}_{0}\right)$ by chemiluminescence microparticle immunoassay.

\section{Quality control}

Three standard substances $(3,6$, and $12 \mathrm{ng} / \mathrm{mL})$ were measured once per week in high, medium, and low order, over a 3-month cycle for quality control. All quality control values were found to be in an effective range, suggesting that the FK506 concentration values of samples were reliable.

\section{DNA extraction and measurement}

DNA was extracted from peripheral venous blood from the KTRs per the manufacturer

Genetics and Molecular Research 15 (3): gmr.15038464 
instructions (WIZARD Genomic DNA purification kit, Beijing Solarbio Bioscience \& Technology Co., Ltd., Beijing, China) for polymerase chain reaction (PCR). Allele-specific PCR was applied to classify the PXR six-base deletion (rs3842689) mutation and the direct sequencing method was used to investigate the genotypes of PXR 7635G $>A$ (rs6785049) and PXR 24381C $>$ A (rs1523127).

\section{Measurement methods}

The PXR six-base deletion gene was detected using the allelic-special touchdown PCR method. Each DNA sample was configured with two tubes: one with a normal upstream primer (F1) and the other with a mutant upstream primer (F2) (Yang et al., 2010). They were then reacted in a PCR system $(25 \mu \mathrm{L})$ containing 2X PCR Master Mix (12.5 $\mu \mathrm{L}$ ) comprising $50 \mathrm{U} / \mathrm{mL}$ Taq DNA polymerase, $400 \mu \mathrm{M}$ deoxynucleoside triphosphates, $3 \mathrm{mM} \mathrm{MgCl}, 1 \mu \mathrm{L}$ upstream and downstream primers $(10 \mu \mathrm{M}), 2 \mu \mathrm{L}$ DNA template, and $8.5 \mu \mathrm{L}$ nuclease-free water. The PCR regimen was as follows: pre-denaturation at $94^{\circ} \mathrm{C}$ for $5 \mathrm{~min} ; 20$ cycles of denaturation at $94^{\circ} \mathrm{C}$ for $30 \mathrm{~s}$, annealing at $62^{\circ} \mathrm{C}$ for $30 \mathrm{~s}$, and extention at $72^{\circ} \mathrm{C}$ for $30 \mathrm{~s}$, in which the annealing temperature decreased by $0.5^{\circ} \mathrm{C}$ per cycle; 20 cycles of denaturation at $94^{\circ} \mathrm{C}$ for $30 \mathrm{~s}$, annealing at $62^{\circ} \mathrm{C}$ for $30 \mathrm{~s}$, and extention at $72^{\circ} \mathrm{C}$ for $30 \mathrm{~s}$; and a final extention at $72^{\circ} \mathrm{C}$ for $5 \mathrm{~min}$. After the paired PCR amplification products had been electrophoresed on $2.5 \%$ agarose gel, the results were analyzed using an ultraviolet gel imaging system.

The PXR 24381C>A mutation gene was reacted using the same PCR system $(25 \mu \mathrm{L})$, and the PCR products were treated in the same way for direct sequencing (de Jonge et al., 2008).

\section{Statistical analysis}

All data were processed using the SPSS19.0 software and are reported as means \pm standard deviation (SD). Differences were considered to be statistically significant if $\mathrm{P}<$ 0.05 . The $t$-test was used to compare the differences in clinical data between each group, and non-normal distribution variables were transformed logarithmically. Enumeration data were subjected to the chi-square test and are reported as N (\%). Logistical regression was applied to analyze risk factors of adverse reactions resulting from FK506.

\section{RESULTS}

\section{PCR amplification primer sequences} in Table 1.

The PCR primer sequences and the lengths of the amplification products are shown

\section{Comparison of general clinical data from patients with different PXR six-base deletion (rs3842689) genotypes}

The general clinical data for the 336 KTRs were compared (Table 2), and no statistical differences were found $(\mathrm{P}>0.05)$.

Genetics and Molecular Research 15 (3): gmr.15038464 
Table 1. Polymerase chain reaction (PCR) amplification primer sequences and lengths of the amplification products.

\begin{tabular}{l|l|l|c}
\hline & Name of primer & Primer sequence & Length of amplification products \\
\hline \multirow{4}{*}{ rs3842689 } & 6-base deletion-F1 & 5'-AATCACCACAGGAGAAGC-3' & $163 \mathrm{bp}$ \\
\cline { 2 - 4 } & 6-base deletion-F2 & 5'-AAACACCACAGCCTTAACT-3' & $169 \mathrm{bp}$ \\
\cline { 2 - 4 } & 6-base deletion-R & 5'-AAAAGGAATCAGGACTGAGT-3' & \\
\hline \multirow{2}{*}{ rs6785049 } & $7635 \mathrm{G}>\mathrm{A}$ & 5'-TAGGTCAGGATTTGTATAGGAGC-3' & \\
\cline { 2 - 4 } & $7635 \mathrm{G}>\mathrm{A}$ & 5'-CCATCCCAGAACCTGCATATC-3' & \\
\hline \multirow{2}{*}{ rs1523127 } & $24381 \mathrm{C}>\mathrm{A}$ & 5'-CCCCTTTTCCTGTGTTTTTG-3' & \\
\cline { 2 - 4 } & $24381 \mathrm{C}>\mathrm{A}$ & 5'-CAACATTAAGTGATTGTTTTCATGC-3' & \\
\hline
\end{tabular}

Table 2. Comparison of clinical data for patients with different PXR rs3842689 genotypes.

\begin{tabular}{l|c|c|c}
\hline & WW $(\mathrm{N}=212)$ & MM $(\mathrm{N}=52)$ & WM $(\mathrm{N}=72)$ \\
\hline Cadaveric kidney (\%) & $161(75.94 \%)$ & $43(82.69 \%)$ & $58(80.56 \%)$ \\
\hline Male/female & $137 / 75$ & $40 / 12$ & $48 / 24$ \\
\hline Age at transplant (years) & $40.35 \pm 10.09$ & $40.58 \pm 12.13$ & $38.89 \pm 10.34$ \\
\hline Time of dialysis (months) & $10.84 \pm 12.07$ & $11.60 \pm 10.86$ & $10.01 \pm 8.55$ \\
\hline Transplant weight $(\mathrm{kg})$ & $57.24 \pm 9.76$ & $56.52 \pm 9.16$ & $57.87 \pm 12.30$ \\
\hline BMI $\left(\mathrm{kg} / \mathrm{m}^{2}\right)$ & $20.58 \pm 2.78$ & $20.47 \pm 2.88$ & $21.03 \pm 2.96$ \\
\hline Treatment course (months) & $38.82 \pm 25.41$ & $36.04 \pm 22.21$ & $33.17 \pm 21.22$ \\
\hline
\end{tabular}

$\mathrm{BMI}=$ body mass index

\section{Influence of PXR six-base deletion (rs3842689) gene polymorphism on FK506 blood trough concentration and $\mathrm{C}_{0} / \mathrm{D}$ value}

Table 3 and Figures 1 and 2 show the influence of the PXR rs3842689 gene polymorphism on FK506 blood trough concentration and the $\mathrm{C}_{0} / \mathrm{D}$ value. At different time points after drug administration, homozygous mutation MM gene carriers had a higher blood trough concentration than wild-type homozygous WW gene carriers, but the difference was not statistically significant $(\mathrm{P}>0.05)$.

\section{Results of PXR 7635G >A (rs6785049) and PXR 24381C >A (rs1523127) genotyping}

The included KTRs were subjected to PXR rs6785049 and rs1523127 genotype detection and the results are shown in Table 4. Of the 336 included patients, 39 had the rs6785049 mutation, with a mutation frequency of $11.61 \%$, and 55 had the rs 1523127 mutation, with a mutation frequency of $16.37 \%$.

\section{Influence of the PXR 7635G>A (rs6785049) gene polymorphism on FK506 blood trough concentration and $C_{0} / D$ value}

Table 5 shows the influence of the PXR 7635G $>$ A (rs6785049) gene polymorphism on FK506 blood trough concentration and $\mathrm{C}_{0} / \mathrm{D}$ value. The PXR 7635G $>\mathrm{A}$ (rs6785049) gene polymorphism did not have any obvious influence on FK506 blood trough concentration or $\mathrm{C}_{0} / \mathrm{D}$ value at the different time points after drug administration $(\mathrm{P}>0.05)$. 
Table 3. Influence of the PXR six-base deletion gene polymorphism on FK506 blood trough concentration and $\mathrm{C}_{0} / \mathrm{D}$ (means $\left.\pm \mathrm{SD}\right)$.

\begin{tabular}{|c|c|c|c|c|}
\hline Time after drug use & $\begin{array}{c}\text { PXR rs3842689 } \\
\text { Genotype }\end{array}$ & Daily dosage $(\mathrm{mg} / \mathrm{kg})$ & $\begin{array}{c}\text { Blood trough } \\
\text { concentration }(\mathrm{ng} / \mathrm{mL})\end{array}$ & $\begin{array}{c}\mathrm{C}_{0} / \mathrm{D} \\
\left(\mathrm{ng} \cdot \mathrm{kg} \cdot \mathrm{mL}^{-1} \cdot \mathrm{mg}^{-1}\right)\end{array}$ \\
\hline \multirow[t]{3}{*}{ Seven days } & WW & $0.06 \pm 0.02$ & $5.88 \pm 3.45$ & $103.26 \pm 75.81$ \\
\hline & MM & $0.06 \pm 0.02$ & $7.04 \pm 3.71$ & $123.72 \pm 69.12$ \\
\hline & WM & $0.08 \pm 0.04$ & $5.70 \pm 3.41$ & $129.76 \pm 74.18$ \\
\hline \multirow[t]{3}{*}{ Fifteen days } & WW & $0.07 \pm 0.04$ & $6.47 \pm 3.29$ & $113.28 \pm 64.27$ \\
\hline & MM & $0.08 \pm 0.02$ & $7.08 \pm 2.75$ & $104.84 \pm 71.40$ \\
\hline & WM & $0.07 \pm 0.04$ & $6.12 \pm 2.52$ & $87.49 \pm 67.56$ \\
\hline \multirow[t]{3}{*}{ One month } & WW & $0.07 \pm 0.04$ & $6.35 \pm 2.74$ & $110.47 \pm 73.43$ \\
\hline & MM & $0.07 \pm 0.04$ & $7.47 \pm 2.87$ & $118.45 \pm 81.76$ \\
\hline & WM & $0.09 \pm 0.04$ & $6.58 \pm 3.09$ & $96.39 \pm 75.48$ \\
\hline \multirow[t]{3}{*}{ Three months } & WW & $0.06 \pm 0.03$ & $6.42 \pm 2.64$ & $135.07 \pm 85.31$ \\
\hline & MM & $0.06 \pm 0.03$ & $6.34 \pm 2.60$ & $110.96 \pm 57.23$ \\
\hline & WM & $0.07 \pm 0.04$ & $6.65 \pm 2.63$ & $117.60 \pm 83.85$ \\
\hline \multirow[t]{3}{*}{ Six months } & WW & $0.05 \pm 0.03$ & $5.87 \pm 2.81$ & $144.88 \pm 103.34$ \\
\hline & MM & $0.06 \pm 0.03$ & $6.59 \pm 2.35$ & $124.74 \pm 76.38$ \\
\hline & WM & $0.06 \pm 0.03$ & $6.37 \pm 2.69$ & $125.17 \pm 76.50$ \\
\hline \multirow[t]{3}{*}{ Twelve months } & WW & $0.06 \pm 0.03$ & $5.76 \pm 2.30$ & $147.37 \pm 86.91$ \\
\hline & MM & $0.06 \pm 0.03$ & $6.15 \pm 2.16$ & $111.03 \pm 51.24$ \\
\hline & WM & $0.06 \pm 0.03$ & $5.42 \pm 1.89$ & $100.99 \pm 51.52$ \\
\hline \multirow[t]{3}{*}{ Twenty-four months } & WW & $0.05 \pm 0.03$ & $5.60 \pm 2.31$ & $147.86 \pm 97.78$ \\
\hline & MM & $0.05 \pm 0.03$ & $6.48 \pm 4.11$ & $130.31 \pm 85.65$ \\
\hline & WM & $0.05 \pm 0.03$ & $5.82 \pm 4.05$ & $129.67 \pm 44.26$ \\
\hline \multirow[t]{3}{*}{ Thirty-six months } & WW & $0.05 \pm 0.03$ & $5.13 \pm 1.87$ & $154.22 \pm 102.13$ \\
\hline & MM & $0.06 \pm 0.02$ & $5.16 \pm 1.86$ & $87.44 \pm 28.83$ \\
\hline & WM & $0.04 \pm 0.03$ & $5.51 \pm 3.04$ & $107.20 \pm 46.89$ \\
\hline \multirow[t]{3}{*}{ Sixty months } & WW & $0.04 \pm 0.03$ & $4.84 \pm 1.71$ & $193.66 \pm 187.00$ \\
\hline & MM & $0.04 \pm 0.03$ & $5.40 \pm 2.11$ & $134.27 \pm 50.43$ \\
\hline & WM & $0.04 \pm 0.03$ & $4.49 \pm 1.47$ & $123.71 \pm 23.94$ \\
\hline
\end{tabular}

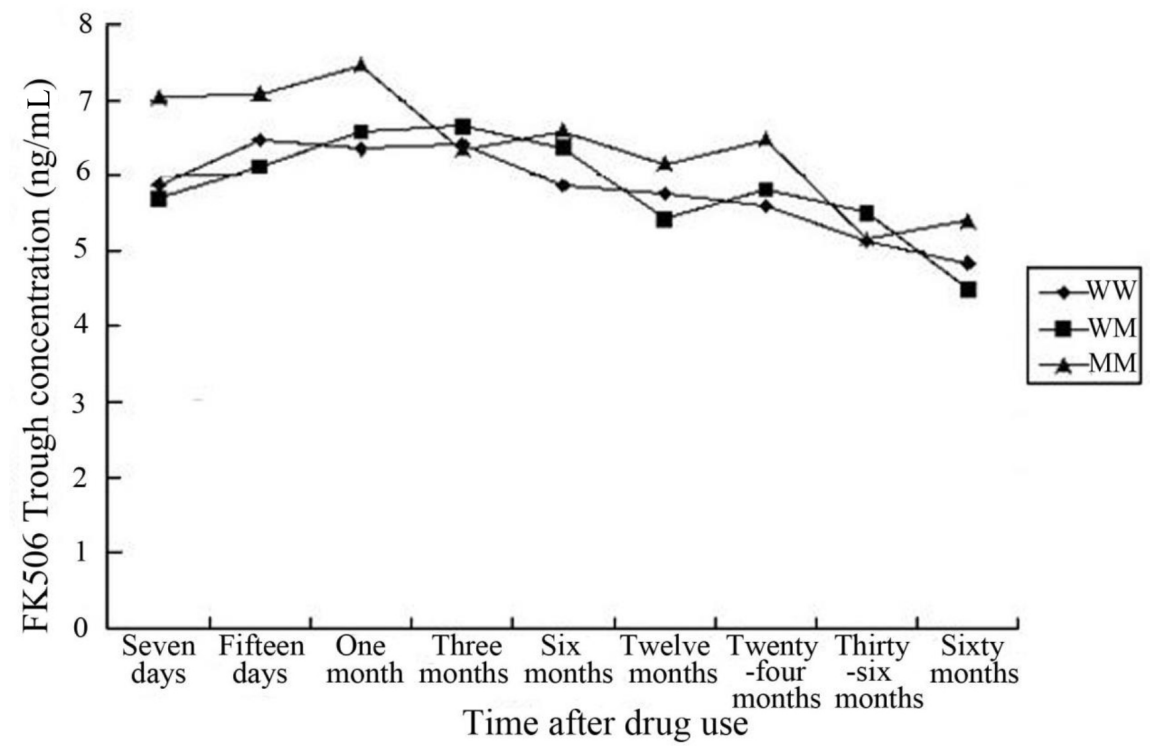

Figure 1. Influence of medication time on FK506 blood trough concentration.

Genetics and Molecular Research 15 (3): gmr.15038464 


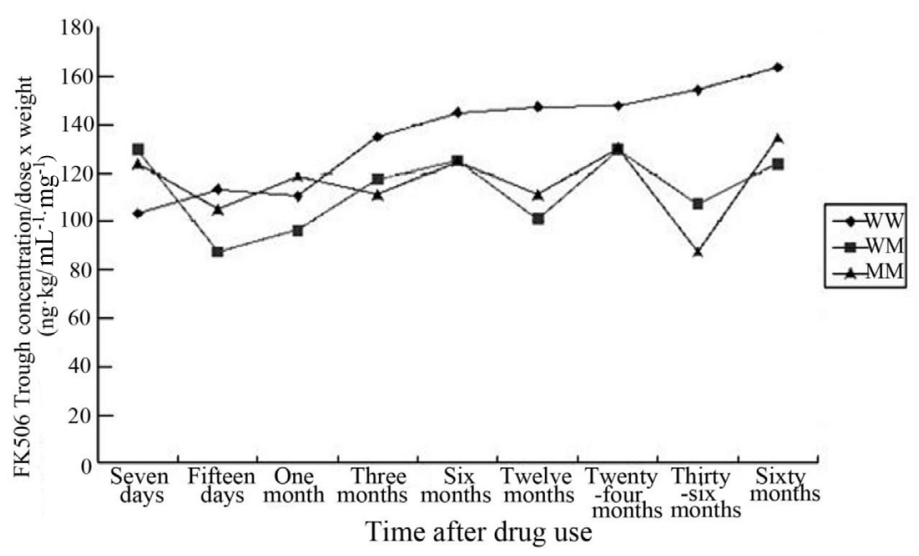

Figure 2. Influence of medication time on $\mathrm{C}_{0} / \mathrm{D}$.

\section{Table 4. PXR rs6785049 and rs1523127 mutation frequencies.}

\begin{tabular}{l|c|c}
\hline Genotypes & Number of cases with mutation & Mutation frequency \\
\hline PXR 7635G $>$ A $($ rs6785049) & 39 & $11.61 \%$ \\
\hline PXR 24381C $>$ A (rs1523127) & 55 & $16.37 \%$ \\
\hline
\end{tabular}

Table 5. Influence of the PXR 7635G $>$ A (rs6785049) gene polymorphism on FK506 blood trough concentration and $\mathrm{C}_{0} / \mathrm{D}$ value (means $\pm \mathrm{SD}$ ).

\begin{tabular}{l|l|c|c|c}
\hline Time after drug use & 7635G $>$ A genotype & $\begin{array}{c}\text { Daily dosage } \\
(\mathrm{mg} / \mathrm{kg})\end{array}$ & $\begin{array}{c}\text { Blood trough } \\
\text { concentration }(\mathrm{ng} / \mathrm{mL})\end{array}$ & $\begin{array}{c}\mathrm{C} / \mathrm{D} \\
\left(\mathrm{ng} \cdot \mathrm{kg} \cdot \mathrm{mL}^{-1} \cdot \mathrm{mg}-1\right)\end{array}$ \\
\hline \multirow{2}{*}{ Seven days } & Mutant type & $0.07 \pm 0.03$ & $5.84 \pm 3.98$ & $103.67 \pm 43.51$ \\
\cline { 2 - 5 } & Primitive type & $0.06 \pm 0.03$ & $6.02 \pm 3.42$ & $110.29 \pm 77.80$ \\
\hline \multirow{2}{*}{ Fifteen days } & Mutant type & $0.07 \pm 0.03$ & $5.93 \pm 3.11$ & $102.79 \pm 69.17$ \\
\cline { 2 - 5 } & Primitive type & $0.07 \pm 0.03$ & $6.50 \pm 3.06$ & $107.45 \pm 71.59$ \\
\hline \multirow{2}{*}{ Three months } & Mutant type & $0.08 \pm 0.03$ & $6.27 \pm 2.57$ & $88.34 \pm 62.26$ \\
\cline { 2 - 5 } & Primitive type & $0.07 \pm 0.03$ & $6.62 \pm 2.89$ & $113.42 \pm 76.48$ \\
\hline \multirow{2}{*}{ Six months } & Mutant type & $0.06 \pm 0.03$ & $6.91 \pm 3.05$ & $140.57 \pm 80.39$ \\
\cline { 2 - 5 } & Primitive type & $0.06 \pm 0.03$ & $6.37 \pm 2.54$ & $126.63 \pm 82.53$ \\
\hline \multirow{2}{*}{ Twelve months } & Mutant type & $0.06 \pm 0.03$ & $6.58 \pm 2.48$ & $159.80 \pm 108.37$ \\
\cline { 2 - 5 } & Primitive type & $0.05 \pm 0.03$ & $6.10 \pm 2.73$ & $122.79 \pm 58.96$ \\
\hline \multirow{2}{*}{ Twenty-four months } & Mutant type & $0.06 \pm 0.03$ & $5.54 \pm 2.20$ & $135.77 \pm 82.81$ \\
\cline { 2 - 5 } & Primitive type & $0.05 \pm 0.03$ & $5.80 \pm 2.22$ & $154.00 \pm 92.03$ \\
\cline { 2 - 5 } & Mutant type & $0.05 \pm 0.03$ & $5.85 \pm 3.29$ & $140.27 \pm 91.07$ \\
\hline \multirow{2}{*}{ Thirty-six months } & Primitive type & $0.05 \pm 0.03$ & $5.77 \pm 2.96$ & $135.29 \pm 81.25$ \\
\cline { 2 - 5 } & Primitive type & $0.05 \pm 0.04$ & $4.64 \pm 1.62$ & $157.21 \pm 100.27$ \\
\hline \multirow{2}{*}{ Sixty months } & Mutant type & $0.04 \pm 0.02$ & $5.32 \pm 2.21$ & $187.64 \pm 133.86$ \\
\cline { 2 - 5 } & Primitive type & $0.05 \pm 0.05$ & $5.06 \pm 1.44$ & $212.04 \pm 210.73$ \\
\hline
\end{tabular}

\section{Influence of the PXR 24381C $>$ A (rs1523127) gene polymorphism on FK506 blood trough concentration and $\mathrm{C}_{0} / \mathrm{D}$ value}

Table 6 shows the influence of the PXR 24381C $>$ A (rs1523127) gene polymorphism on FK506 blood trough concentration and $\mathrm{C}_{0} / \mathrm{D}$ value. After 3 months of medication, the blood 
trough concentration was higher in the mutant individuals than in the wild-type individuals $(\mathrm{P}=0.030)$, and the mutant individuals had a markedly higher $\mathrm{C}_{0} / \mathrm{D}$ value than the wild-type individuals did after 6 months of medication $(\mathrm{P}=0.009)$. There were no significant differences in blood trough concentration or $\mathrm{C}_{0} / \mathrm{D}$ value at the other time points $(\mathrm{P}>0.05)$.

\begin{tabular}{|c|c|c|c|c|}
\hline Time after drug use & $\begin{array}{l}24381 \mathrm{C}>\mathrm{A} \\
\text { genotype }\end{array}$ & Daily dosage (mg/kg) & Blood trough concentration $(\mathrm{ng} / \mathrm{mL})$ & $\mathrm{C}_{0} / \mathrm{D}\left(\mathrm{ng} \cdot \mathrm{kg} \cdot \mathrm{mL}^{-1} \cdot \mathrm{mg}^{-1}\right)$ \\
\hline \multirow[t]{2}{*}{ Seven days } & Mutant type & $0.07 \pm 0.03$ & $6.37 \pm 3.48$ & $127.42 \pm 84.06$ \\
\hline & Primitive type & $0.06 \pm 0.03$ & $5.95 \pm 3.49$ & $107.24 \pm 73.85$ \\
\hline \multirow[t]{2}{*}{ Fifteen days } & Mutant type & $0.07 \pm 0.03$ & $6.41 \pm 2.96$ & $107.37 \pm 81.99$ \\
\hline & Primitive type & $0.07 \pm 0.03$ & $6.50 \pm 3.11$ & $106.55 \pm 69.32$ \\
\hline \multirow[t]{2}{*}{ One month } & Mutant type & $0.07 \pm 0.03$ & $6.48 \pm 2.93$ & $104.28 \pm 73.90$ \\
\hline & Primitive type & $0.07 \pm 0.03$ & $6.57 \pm 2.84$ & $109.28 \pm 75.06$ \\
\hline \multirow[t]{2}{*}{ Three months } & Mutant type & $0.06 \pm 0.03$ & $6.77 \pm 3.34$ & $146.71 \pm 89.42$ \\
\hline & Primitive type & $0.06 \pm 0.03$ & $6.40 \pm 2.41$ & $125.70 \pm 80.52$ \\
\hline \multirow[t]{2}{*}{ Six months } & Mutant type & $0.06 \pm 0.03$ & $6.75 \pm 2.71$ & $184.20 \pm 142.69$ \\
\hline & Primitive type & $0.05 \pm 0.03$ & $6.00 \pm 2.73$ & $129.83 \pm 81.95$ \\
\hline \multirow[t]{2}{*}{ Twelve months } & Mutant type & $0.05 \pm 0.03$ & $6.18 \pm 2.32$ & $155.87 \pm 85.56$ \\
\hline & Primitive type & $0.05 \pm 0.03$ & $5.63 \pm 2.19$ & $129.10 \pm 77.88$ \\
\hline \multirow[t]{2}{*}{ Twenty-four months } & Mutant type & $0.04 \pm 0.03$ & $5.62 \pm 4.23$ & $152.74 \pm 64.61$ \\
\hline & Primitive type & $0.05 \pm 0.03$ & $5.18 \pm 2.20$ & $141.61 \pm 94.17$ \\
\hline \multirow[t]{2}{*}{ Thirty-six months } & Mutant type & $0.03 \pm 0.03$ & $5.22 \pm 1.51$ & $193.75 \pm 88.97$ \\
\hline & Primitive type & $0.05 \pm 0.03$ & $5.23 \pm 2.26$ & $148.22 \pm 97.14$ \\
\hline \multirow[t]{2}{*}{ Sixty months } & Mutant type & $0.02 \pm 0.01$ & $4.35 \pm 1.62$ & $212.90 \pm 95.66$ \\
\hline & Primitive type & $0.05 \pm 0.03$ & $4.83 \pm 1.77$ & $202.83 \pm 217.40$ \\
\hline
\end{tabular}

\section{Distribution of the PXR gene polymorphism in the control group and the groups with different adverse reactions}

The data for the PXR gene polymorphism are shown in Table 7. The PXR rs3842689 and rs6785049 genotypes were distributed in each group without statistical significance $\left(\chi^{2}\right.$ $\left.=8.364, \mathrm{P}=0.756 ; \chi^{2}=6.273, \mathrm{P}=0.393\right)$. There were statistically significant differences in PXR rs1523127 genotype distribution between the various groups $\left(\chi^{2}=13.126, \mathrm{P}=0.041\right)$ and the PXR rs1523127 mutation increased in the hyperlipidemia group in comparison with the control group.

Table 7. Relationship between the PXR genotype and adverse reactions resulting from FK506.

\begin{tabular}{l|c|c|c|c|c|c|c|c}
\hline & $\begin{array}{c}\text { Control group } \\
(203 \text { cases) }\end{array}$ & $\begin{array}{c}\text { Liver injury } \\
\text { group }(8 \text { cases) }\end{array}$ & $\begin{array}{c}\text { Hyperglycemia } \\
\text { group } \\
(34 \text { cases })\end{array}$ & $\begin{array}{c}\text { Hematotoxicity } \\
\text { group (32 cases) }\end{array}$ & $\begin{array}{c}\text { Hyperlipidemia } \\
\text { group (30 cases) }\end{array}$ & $\begin{array}{c}\text { Hypertension } \\
\text { group (14 cases) }\end{array}$ & $\begin{array}{c}\text { Gastrointestinal } \\
\text { reaction group } \\
(15 \text { cases) }\end{array}$ & $\begin{array}{c}\text { Total adverse } \\
\text { reaction } \\
(133 \text { cases })\end{array}$ \\
\hline $\begin{array}{l}\text { rs3842689 } \\
\text { Primitive type }\end{array}$ & $126(62.07 \%)$ & $8(100 \%)$ & $21(61.76 \%)$ & $20(62.50 \%)$ & $21(70 \%)$ & $9(64.29 \%)$ & $8(53.33 \%)$ & $87(65.41 \%)$ \\
\hline $\begin{array}{l}\text { Mutation } \\
\text { homozygote }\end{array}$ & $32(15.76 \%)$ & $0(0 \%)$ & $6(17.65 \%)$ & $6(18.75 \%)$ & $3(10 \%)$ & $0(0 \%)$ & $4(26.67 \%)$ & $19(14.28 \%)$ \\
\hline $\begin{array}{l}\text { Mutation } \\
\text { heterozygote }\end{array}$ & $45(22.17 \%)$ & $0(0 \%)$ & $7(20.59 \%)$ & $6(18.75 \%)$ & $6(20 \%)$ & $5(35.71 \%)$ & $3(20 \%)$ & $27(20.31 \%)$ \\
\hline $\begin{array}{l}\text { rs6785049 } \\
\text { Primitive type }\end{array}$ & $175(86.21 \%)$ & $8(100 \%)$ & $32(94.12 \%)$ & $28(87.50 \%)$ & $29(96.67 \%)$ & $10(71.43 \%)$ & $14(93.33 \%)$ & $121(90.98 \%)$ \\
\hline Mutant type & $28(13.79 \%)$ & $0(0 \%)$ & $2(5.88 \%)$ & $4(12.50 \%)$ & $1(3.33 \%)$ & $4(28.57 \%)$ & $1(6.67 \%)$ & $12(9.02 \%)$ \\
\hline $\begin{array}{l}\text { rs1523127 } \\
\text { Primitive type }\end{array}$ & $171(84.24 \%)$ & $7(87.50 \%)$ & $29(85.29 \%)$ & $31(96.88 \%)$ & $20(66.67 \%)$ & $10(71.43 \%)$ & $15(100 \%)$ & $111(83.46)$ \\
\hline Mutant type & $32(15.76 \%)$ & $1(12.50 \%)$ & $5(14.71 \%)$ & $1(3.12 \%)$ & $10(33.33 \%)$ & $4(28.57 \%)$ & $0(0 \%)$ & $22(16.54)$ \\
\hline
\end{tabular}




\section{Logistical regression}

According to the regression results, the rs6785049 and rs1523127 genotypes had no obvious correlation with adverse reactions resulting from FK506 $(\mathrm{P}>0.05)$. Logistical regression revealed that PXR rs3842689 patients with the wild-type homozygote WW genotype were 15.8 times more likely to suffer from gastrointestinal reaction after medication compared with mutation homozygote MM genotype individuals.

\section{DISCUSSION}

Because it is a key transcriptional regulation factor, PXR participates in the inducible expression of a variety of drug metabolic enzymes and transport proteins, there by indirectly affecting FK506 drug metabolism and transport. At present, international research on NR1I2 gene polymorphisms focuses only on African Americans and Caucasians, instead of Asians or Chinese. Moreover, NR1I2 mutations differ greatly between the races (Tsuchiya et al., 2004; Wang et al., 2007; Swart and Dandara, 2014).

Uno et al. (2003) first discovered the NR1I2 promoter region six-base pair deletion mutation (rs3842689, -/GAGAAG), which occurs in a potential liver nuclear factor (HNF1) binding site. Research indicated that the Hpar-2 promoter activity of HepG2 cells was essentially lost with the six-base deletion mutation, and the PXR six-base deletion mutation caused a decrease in PXR mRNA expression, there by significantly reducing the expression of its target genes CYP3A4 and MDR1 (Liu et al., 2009). Lamba et al. (2008) also discovered an obvious decrease in CYP3A4 expression resulting from six-base deletions under base-induced conditions. Of the included $336 \mathrm{KTRs}$, the frequency of the PXR six-base deletion mutation was $26.07 \%$. Homozygous MM gene carriers had a higher blood trough concentration than native homozygous WW gene carriers at the different time points after medication, but the differences were not statistically significant. Compared with homozygous MM genotype individuals, the risk of native homozygous WW genotype patients developing gastrointestinal reactions increased 15.8 times after medication $(95 \% \mathrm{CI}=10.07-24.80 ; \mathrm{P}=0.000)$. PXR G7635A is mainly associated with CYP3A4 in the intestinal tract (Zhang et al., 2001). Press et al. (2009) found that gene polymorphism in the locus affected the clearance rate of FK506 and the clearance rates of A allele gene carriers and GG genotype patients were $3.9 \pm 0.3$ and $5.4 \pm 0.6 \mathrm{~L} / \mathrm{h}$, respectively. Applying the direct sequencing PCR method, Wang et al. (2013) carried out PXR-C24381A genotyping on 60 patients who were given an oral administration of FK506 after renal transplantation, and the results suggested that the $\mathrm{C}_{0} / \mathrm{D}$ value in the C/A and $\mathrm{C} / \mathrm{C}$ genotype patients was higher than in the $\mathrm{A} / \mathrm{A}$ genotype patients, which suggested that the A/A genotype patients required a higher dosage of FK506 than the C/A and C/C genotype patients to obtain a similar blood trough concentration. Moreover, the PXR six-base deletion (rs3842689), and the PXR 7635G $>$ A (rs6785049) and PXR 24381C $>$ A (rs1523127) gene polymorphisms had no influence on FK506 blood trough concentration or $\mathrm{C}_{0} / \mathrm{D}$ value. Logistical regression revealed that the PXR rs3842689 native homozygous WW genotype was a risk factor that resulted in gastrointestinal reactions but the other genotypes had no significant effects on adverse reactions resulting from FK506.

There are some caveats in this study, which could not be addressed owing to certain limitations with respect to experimental conditions; currently, we are in the process of making the necessary improvements to rectify these limitations.

Genetics and Molecular Research 15 (3): gmr.15038464 
This study aimed to investigate the effect of PXR polymorphisms on tacrolimus blood concentrations and the resulting adverse reactions in KTRs to provide a reliable basis for further studies.

\section{Conflicts of interest}

The authors declare no conflict of interest.

\section{ACKNOWLEDGMENTS}

We would like to express our sincere gratitude to all the researchers involved in this study.

\section{REFERENCES}

Benkali K, Prémaud A, Picard N, Rérolle JP, et al. (2009). Tacrolimus population pharmacokinetic-pharmacogenetic analysis and Bayesian estimation in renal transplant recipients. Clin. Pharmacokinet. 48: 805-816. http://dx.doi. org/10.2165/11318080-000000000-00000

de Jonge H, Naesens M, Vanrenterghem Y and Kuypers DR (2008). The pregnane X receptor (NR1I2) C-25385T single nucleotide polymorphism (SNP) does not affect tacrolimus pharmacokinetics in a large cohort of renal transplant recipients. Ann. Transplant. 13: 33-33.

Elens L, Capron A, Kerckhove VV, Lerut J, et al. (2007). 1199G $>$ A and 2677G $>$ T/A polymorphisms of ABCB1 independently affect tacrolimus concentration in hepatic tissue after liver transplantation. Pharmacogenet. Genomics 17: 873-883.http://dx.doi.org/10.1097/FPC.0b013e3282e9a533

Fei WL, Chen JQ, Zhong SL, Liu YM, et al. (2006). The pharmacokinetics of FK506 and its nanoparticles in aqueous humor of rabbits. Zhonghua Yan Ke Za Zhi 42: 305-308.

Hesselink DA, van Schaik RH, van der Heiden IP, van der Werf M, et al. (2003). Genetic polymorphisms of the CYP3A4, CYP3A5, and MDR-1 genes and pharmacokinetics of the calcineurin inhibitors cyclosporine and tacrolimus. Clin. Pharmacol. Ther. 74: 245-254. http://dx.doi.org/10.1016/S0009-9236(03)00168-1

Lamba J, Lamba V, Strom S, Venkataramanan R, et al. (2008). Novel single nucleotide polymorphisms in the promoter and intron 1 of human pregnane X receptor/NR1I2 and their association with CYP3A4 expression. Drug Metab. Dispos. 36: 169-181. http://dx.doi.org/10.1124/dmd.107.016600

Liu Y, Ji W, Yin Y, Fan L, et al. (2009). The effects of splicing variant of PXR PAR-2 on CYP3A4 and MDR1 mRNA expressions. Clin. Chim. Acta 403: 142-144.http://dx.doi.org/10.1016/j.cca.2009.02.006

Miura M, Satoh S, Inoue K, Kagaya H, et al. (2008). Influence of CYP3A5, ABCB1 and NR1I2 polymorphisms on prednisolone pharmacokinetics in renal transplant recipients. Steroids 73: 1052-1059. http://dx.doi.org/10.1016/j. steroids.2008.04.002

Press RR, Ploeger BA, den Hartigh J, van der Straaten T, et al. (2009). Explaining variability in tacrolimus pharmacokinetics to optimize early exposure in adult kidney transplant recipients. Ther. Drug Monit. 31: 187-197. http://dx.doi. org/10.1097/FTD.0b013e31819c3d6d

Qi YJ, Li GF, Zhou Y, Wang MM, et al. (2012). Determination of FK506 in rat blood by LC-MS/MS and effect of silymarin on its pharmacokinetics. J. Liq. Chromatogr. Relat. Technol. 35: 2217-2227.

Swart M and Dandara C (2014). Genetic variation in the 3'-UTR of CYP1A2, CYP2B6, CYP2D6, CYP3A4, NR1I2, and UGT2B7: potential effects on regulation by microRNA and pharmacogenomics relevance. Front. Genet. 5: 167. http://dx.doi.org/10.3389/fgene.2014.00167

Tsuchiya N, Satoh S, Tada H, Li Z, et al. (2004). Influence of CYP3A5 and MDR1 (ABCB1) polymorphisms on the pharmacokinetics of tacrolimus in renal transplant recipients. Transplantation 78: 1182-1187. http://dx.doi. org/10.1097/01.TP.0000137789.58694.B4

Uno Y, Sakamoto Y, Yoshida K, Hasegawa T, et al. (2003). Characterization of six base pair deletion in the putative HNF1-binding site of human PXR promoter. J. Hum. Genet. 48: 594-597. http://dx.doi.org/10.1007/s10038-003$\underline{0076-5}$

Wang CY, Xu X, Li MC, Li Q, et al. (2015a). Analysis of tacrolimus blood concentrations in renal transplant patients. Genet. Mol. Res. 14: 3791-3797. http://dx.doi.org/10.4238/2015.April.22.8

Genetics and Molecular Research 15 (3): gmr.15038464 
Wang J, Li P-H, Wen L and Lu L (2013). Guidance for individual administration of tacrolimus in kidney-transplanted patients according to pregnane X receptor genetic polymorphism. China Pharmacy 22: 2049-2050.

Wang L, Liu LH, Tong WH, Wang MX, et al. (2015b). Effect of CYP3A5 gene polymorphisms on tacrolimus concentration/ dosage ratio in adult liver transplant patients. Genet. Mol. Res. 14: 15148-15157. http://dx.doi.org/10.4238/2015. November.25.3

Wang XD, Li JL, Su QB, Deng XY, et al. (2007). A pharmacogenetic study of pregnane X receptor (NR1I2) in Han Chinese. Curr. Drug Metab. 8: 778-786. http://dx.doi.org/10.2174/138920007782798199

Yang L, Ge W, Yu F and Zhu H (2010). Impact of VKORC1 gene polymorphism on interindividual and interethnic warfarin dosage requirement - a systematic review and meta analysis. Thromb. Res. 125: e159-e166. http://dx.doi. org/10.1016/j.thromres.2009.10.017

Yuan J, Zhai JJ, Huang X, Zhou SY, et al. (2012). Ocular safety and pharmacokinetics study of FK506 suspension eye drops after corneal transplantation. J. Ocul. Pharmacol. Ther. 28: 153-158. http://dx.doi.org/10.1089/jop.2011.0108

Zhang J, Kuehl P, Green ED, Touchman JW, et al. (2001). The human pregnane X receptor: genomic structure and identification and functional characterization of natural allelic variants. Pharmacogenetics 11: 555-572. http://dx.doi. org/10.1097/00008571-200110000-00003

Genetics and Molecular Research 15 (3): gmr.15038464 